\title{
Structural Dynamics of Nanoalloy Catalysts inside Fuel Cells by Combined in operando X- ray Spectroscopy and Total Scattering
}

\author{
Valeri Petkov petko1vg@cmich.edu
}

\author{
Department of Physics, Central Michigan University, Mt. Pleasant, MI 48859
}

Many catalysts for energy related applications, in particular metallic nanoalloys, readily undergo atomiclevel changes during electrochemical reactions. The origin, dynamics and implications of the changes for the performance of the catalysts inside operating devices though are not well understood. This is largely because they are studied on model nanocatalysts under controlled laboratory conditions. We will present results from a study on the dynamic behavior of nanoalloys of Pt with $3 d$-transition metals as they function as catalysts for the oxygen reduction reaction at the cathode of an operating proton exchange membrane fuel cell (PEMFC). The study involves combined synchrotron x-ray spectroscopy and total scattering allowing to characterize the catalysts with atomic-level precision $(\sim 0.02 \AA)$ and element specificity $(\sim 2-3$ at. \%) in both time $(\sim 1 \mathrm{~min})$ and space $(\sim \mu \mathrm{m})$ resolved manner. ${ }^{1}$ We find that the nanoalloys change profoundly, from the initial pristine state to the catalytically active form and further along the cell operation. The rate and magnitude of the changes may be rationalized when the limits of traditional relationships used to connect the composition and structure of nanoalloy catalysts with their activity and stability, such as Vegard's law, are recognized. We will also show how knowledge about the atomic-level evolution of nanoalloy catalysts during their lifetime inside operating devices inspires new efforts to stabilize structure states that are beneficial to their activity and stability.
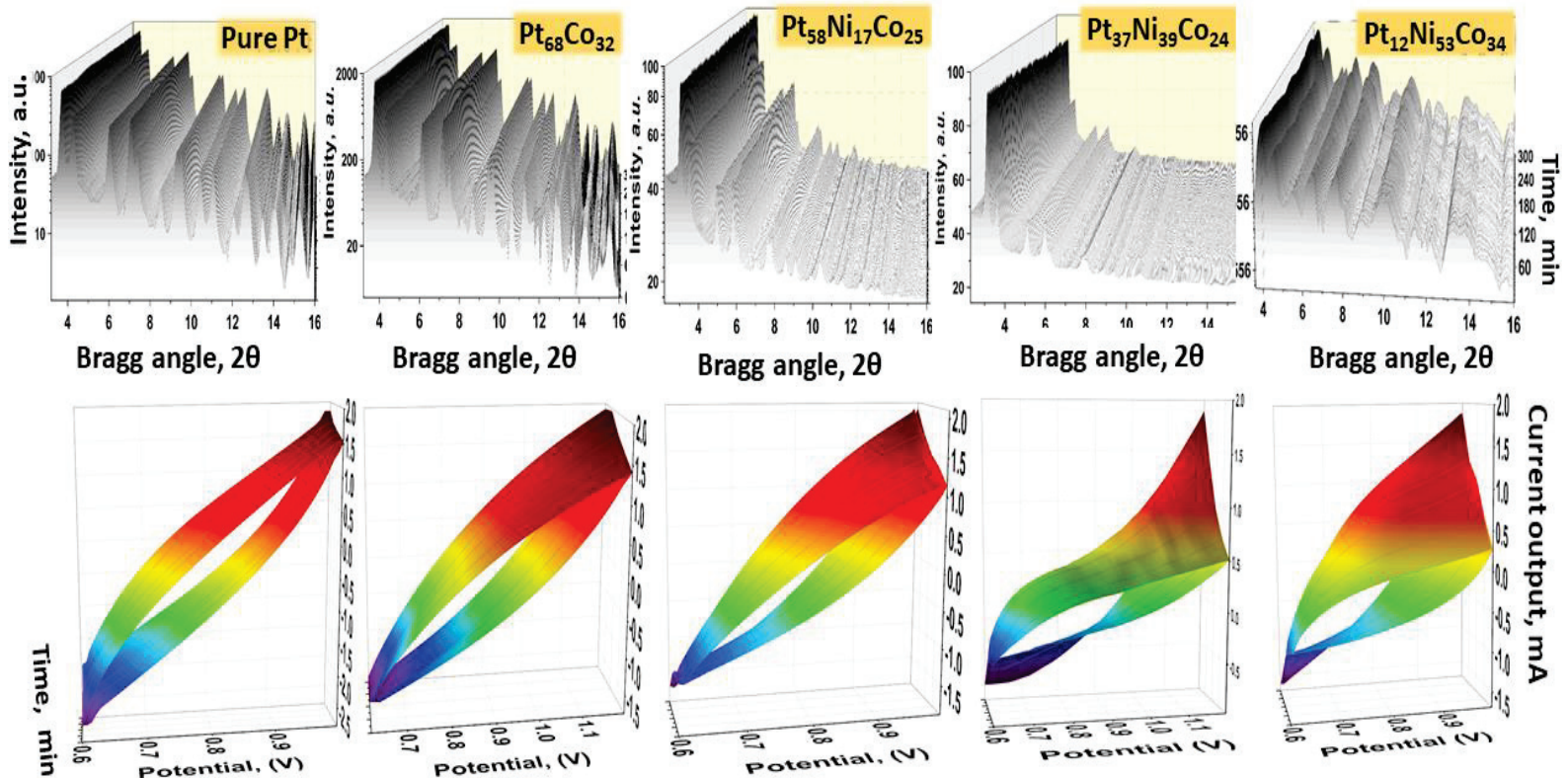

Figure. (top pannel) In operando XRD patterns for Pt-based nanoalloys used as catalysts at the cathode of a PEMFC. The patterns are collected during $5 \mathrm{~h}$ of the PEMFC operation in 3 min intervals. Also shown are the concurrently collected polarization curves representing the PEMFC current output resulted from the repetitive application of external voltage in the range from $0.6 \mathrm{~V}$ to $1.0 \mathrm{~V}$.

1. V. Petkov et al., Nanoscale, 2019, 11, 5512-5525. 